\title{
USO DA INTELIGÊNCIA ARTIFICIAL NO DIAGNÓSTICO E ELEGIBILIDADE DE PACIENTES PARA CUIDADOS PALIATIVOS: \\ LIMITES ÉTICOS E JURÍDICOS
}

\author{
APPLICATIONS OF ARTIFICIAL INTELLIGENCE IN THE DIAGNOSIS AND \\ PATIENT ELIGIBILITY FOR PALLIATIVE CARE: LEGAL AND ETHICAL \\ BOUNDARIES
}

Phillipe Werneque de Oliveira Dias ${ }^{1}$

\begin{abstract}
RESUMO
A Inteligência Artificial se apresenta como uma das maiores transformações da sociedade contemporânea. Embora o alcance de suas aplicações ainda esteja sendo descoberto, existem atualmente vários casos de uso para diagnóstico médico, passando pela detecção de melanomas até o cálculo de probabilidade de morte de um paciente ao dar entrada no hospital. Essas aplicações podem melhorar a qualidade de vida dos pacientes, sendo decisivas para definir, por exemplo, quando a equipe de cuidados paliativos deve ser acionada. Analisamos as questões ético-jurídicas decorrentes da adoção dessas novas práticas tendo como base: os riscos inerentes ao desenvolvimento e adoção da Inteligência Artificial, a Autonomia do Paciente, o Autodiagnóstico e as possíveis ramificações na biopolítica. A metodologia utilizada apresenta um ensaio construído através de pesquisa documental, exibindo soluções disruptivas de uso da Inteligência Artificial na área médica, analisando e problematizando as questões éticas e jurídicas da adoção de tais tecnologias.
\end{abstract}

Palavras chave: Inteligência Artificial, Biodireito e Tecnologia, Cuidados Paliativos.

\begin{abstract}
Artificial Intelligence presents itself as one of the greatest transformations of contemporary society. Although the scope of its applications is still being discovered, there are currently several use cases for medical diagnosis, ranging from melanoma detection to a patient's likelihood of death after checking into a hospital. These applications can improve patients quality of life and drive the decisions, for example, when the palliative care team should be called. We analyze the ethical-legal issues arising from the adoption of these new practices based on the inherent risks in the development and adoption of Artificial Intelligence, Patient Autonomy, Self Diagnosis and the possible ramifications in biopolitics. The methodology used presents an essay built through documentary research, showing disruptive solutions for the use of Artificial Intelligence in the medical area, analyzing and questioning the ethical and legal issues of the adoption of such technologies.
\end{abstract}

Keywords: Artificial Intelligence, Biolaw and Technology, Palliative Care.

1 Graduando em Direito, Faculdade Nacional de Direito, UFRJ. Técnico em Informática e Automação Industrial, CEFET-MG.phiwod@gmail.com 


\section{INTRODUÇÃO}

A Inteligência Artificial é, sem dúvida, o maior avanço na transformação digital que a sociedade contemporânea está vivendo. Desde pequenas funções automatizadas nos celulares, até carros que não precisam de motorista, a tecnologia tem ultrapassado sua posição de facilitadora para assumir uma posição na tomada de decisões, muitas vezes com pouca ou nenhuma interferência humana. No segmento de saúde também começam a despontar os primeiros resultados consistentes no uso da Inteligência Artificial tanto no diagnóstico ${ }^{2}$ quanto no prognóstico de pacientes.

Preliminarmente, importa informar que o termo Inteligência Artificial é aqui usado como gênero das inovações em computação capazes de ações e/ou resultados autônomos, incluindo, portanto, as redes neurais, o aprendizado de máquina, o aprendizado profundo, os processadores de linguagem natural, entre outros, além da própria Inteligência Artificial em sentido estrito, que é definida tradicionalmente como a capacidade (ou tentativa) de fazer com que a tecnologia tenha algum tipo de comportamento humano.

Analisamos aqui as relações jurídicas decorrentes do uso da Inteligência Artificial no diagnóstico e prognóstico de pacientes, separando, por escolha didática, os desafios éticos da adoção de I.A. na saúde, onde enfrentamos novos dilemas sobre a responsabilidade do profissional de saúde frente a uma decisão ou recomendação de máquina, avançando para a análise da autonomia do paciente e as novas opções de autodiagnóstico, onde são trazidas provocações sobre a autonomia do paciente frente a recusa de tratamento e os prováveis usos da Inteligência Artificial na biopolítica e, por fim, analisamos essas novas relações dentro do ordenamento jurídico atual, com destaque para a nova Lei Geral de Proteção de Dados Pessoais ${ }^{3}$ - LGDP, para entender se estamos prontos para lidar com os futuros litígios que irão surgir.

\section{QUANDO A INTELIGÊNCIA ARTIFICIAL É CAPAZ DE DECIDIR}

O uso de ferramentas tecnológicas durante o processo de diagnóstico é bastante comum, sendo inclusive utilizado por muitos organismos internacionais como a OCDE como parâmetro para avaliar o acesso à saúde. Segundo o Mapa Assistencial da Saúde Suplementar de 2018 da

Alguns exemplos, como o chatbot capaz de reconhecer vários tipos de doenças com base nos sintomas fornecidos pelo usuário, podem ser encontrados no portal: https://emerj.com/ai-sector-overviews/ machinelearning-medical- diagnostics-4-current-applications/

3 Brasil. Lei 13709/2018. Disponível em: http://www.planalto.gov.br/ccivil_03/_ato2015-2018/2018 /lei/L13709.htm 
ANS, no Brasil são realizadas 158 tomografias computadorizadas e 169 ressonâncias magnéticas por habitante ${ }^{4}$, números que aproximam o Brasil de países como o Canadá e a Alemanha $^{5}$ em utilização desses equipamentos. Contudo, o uso da tecnologia sempre esteve condicionado ao aval dos profissionais de saúde capacitados para tal ação, sejam os médicos, no caso dos exames por imagem ou exames médicos, ou médicos e bioquímicos nos exames laboratoriais. Dessa forma, mesmo que seja possível ao equipamento fornecer um dado exato, como, por exemplo, a contagem de plaquetas do sangue, o uso dessa informação é condicionada a avaliação e aprovação de um profissional qualificado, que conhece a metodologia utilizada pelo equipamento, podendo repetir o exame, avaliar com outra técnica e até mesmo, na maioria dos casos, repetir o procedimento de forma manual.

A entrada da Inteligência Artificial neste cenário apresenta-se com uma série de vantagens e desafios, por um lado é possível comparar, associar e cruzar dados históricos de milhares de pacientes para obter um diagnóstico, na outra face, é improvável que seja possível a qualquer pessoa, mesmo aos desenvolvedores originais do sistema, compreender o método utilizado para a obtenção de um diagnóstico específico. Isso porque qualquer programa de inteligência artificial é baseado em aprendizado contínuo, ou seja, seus resultados podem ser diferentes com o passar do tempo e com a análise de mais casos, é como se o programa de computador pudesse adquirir experiência e se especializar naquela função, o que nos leva a perguntar: Podemos confiar no diagnóstico fornecido por Inteligência Artificial para decidir o tratamento de um paciente?

Uma equipe multidisciplinar da Universidade de Stanford publicou em $2017^{6}$ o resultado de um experimento onde a Inteligência Artificial através de Aprendizagem Profunda foi utilizada para determinar, durante a admissão dos pacientes no hospital, a probabilidade de morte nos próximos 12 (doze) meses, possibilitando assim recomendações para o encaminhamento de cuidados paliativos. A relevância desse estudo enfrentou um problema central no tratamento de pacientes com doenças graves, onde por um lado temos o desejo crescente por melhor qualidade na terminalidade da vida e, por outro, o desejo de cura, que pode levar a uma demora no encaminhamento do paciente para cuidados paliativos, sacrificando a qualidade de vida. $\mathrm{O}$ resultado culminou em um grupo de pacientes reportado

4 ANS - Agência Nacional de Saúde (2018). Mapa Assistencial da Saúde Suplementar 2018, pags 14 e 15. Disponível em: http://www.ans.gov.br/ images/stories/noticias/pdf/Mapa_Assistencial_2018.pdf

5 OECD Data (2017). Health care use. Disponível em: https://data.oecd.org/healthcare/computed- tomography -ct-exams.htm

6 Anand Avati et al (2017). Improving Palliative Care with Deep Learning. Disponível em: https://arxiv.org/abs/1711.06402 
com $90 \%$ de precisão para a probabilidade de morte em até 12 meses, esses pacientes foram submetidos a análise dos profissionais da equipe de cuidados paliativos do hospital e todos foram considerados elegíveis ao processo.

$\mathrm{Na}$ Universidade de Heidelberg, na Alemanha, foi conduzido um estudo comparativo ${ }^{7}$ entre uma rede de aprendizado profundo e um grupo de 58 dermatologistas, o sistema de Inteligência Artifical e o grupo de médicos deveria identificar melanomas através de imagens dermatoscópicas. $\mathrm{O}$ estudo foi dividido em duas fases, na primeira, os médicos não tinham acesso aos dados dos pacientes, o que resultou num percentual de acurácia de $86.6 \%$ para os médicos, contra $98.8 \%$ para a Inteligência Artificial. No segunda etapa, os médicos receberam informações dos pacientes, o que elevou a acurácia do grupo para $88.9 \%$, ficando ainda bem abaixo dos $98.6 \%$ atingidos pela I.A.

O IEEE - Institute of Electrical and Electronics Engineers mantém um placar online ${ }^{8}$ de estudos acadêmicos onde médicos e Inteligência Artificial tiveram seus resultados comparados, há resultados positivos para a Inteligência Artificial tanto em diagnósticos, como casos de Alzheimer, autismo e mutação de tumores, quanto em procedimentos, como cortes cirúrgicos. Já os médicos se deram melhor na análise de ecocardiogramas e principalmente na análise de imagens hackeadas, onde a mudança de alguns pixels da imagem foi suficiente para fazer com que a classificação por I.A. fosse incorreta, mas não enganou os médicos ${ }^{9}$.

A proliferação desses estudos nos permite acreditar que a adoção da Inteligência Artificial deve ultrapassar a posição que a tecnologia atualmente ocupa na área médica, se até então a tecnologia era ferramenta para obtenção de informação para os diagnósticos médicos, agora a I.A. é quem efetua o próprio diagnóstico e sugere os encaminhamentos.

\section{DESAFIOS ÉTICOS DAS DECISÕES EM SAÚDE POR INTELIGÊNCIA ARTIFICIAL}

Considerando que o assunto é bastante recente e ainda em desenvolvimento, inúmeros são os dilemas éticos e jurídicos sensíveis que irão surgir para enfrentarmos. Como base para este estudo, analisaremos em tópicos alguns pontos destacados pelo Nuffield Council on

7 H A Haenssle et al (2018). Man against machine: diagnostic performance of a deep learning convolutional neural network for dermoscopic melanoma recognition in comparison to 58 dermatologists. Disponível em: https://academic.oup.com/annonc/article/29/8/1836/5004443

8 IEEE Spectrum (2018). AI vs Doctors. Disponível em: https://spectrum.ieee.org/static/ai-vs-doctors

9 Sobre essa importante constatação falamos com mais detalhes no capítulo seguinte, quando discutimos o potencial da I.A. de tomar decisões erradas. 
Bioethics ${ }^{10}$ que deveriam ser debatidos e equacionados na adoção da Inteligência Artificial nos cuidados em saúde.

\section{O POTENCIAL DA I.A. DE TOMAR DECISÕES ERRADAS}

O potencial de uso da Inteligência Artificial ainda não encontrou limites e provavelmente nunca encontrará, grandes companhias investem nesta linha e estão obtendo resultados cada vez mais impressionantes, contudo, nem sempre os resultados são positivamente impressionantes, isso porque, essencialmente, qualquer algoritmo de Inteligência Artificial aprende com base num grupo de dados, também conhecido como amostra de treinamento, após treinada, a I.A. irá analisar os novos dados e adicionar a sua base de conhecimento, melhorando suas decisões. Caso esses dados de treinamento não sejam cuidadosamente tratados, o resultado poderá ser desastroso, é o caso do robô criado pela Microsoft em 2016 para conversar com usuários através da rede social Twitter ${ }^{11}$, que aprendeu a responder aos usuários com afirmações contendo todo tipo de preconceito e palavras de baixo calão. A mesma foi desligada em menos de 24 horas, mas já tinha compartilhado quase 100.000 (cem mil) mensagens, embora nem todas tenham conteúdo ofensivo. Fato semelhante ocorre nas técnicas de reconhecimento facial computadorizado, embora a maioria dos fornecedores prometa uma acurácia quase perfeita, chegando a afirmar que a chance de erro é uma em um milhão ${ }^{12}$, quando o uso é elevado a algo mais concreto e ligado a direitos fundamentais como a saúde ou liberdade, grandes empresas estão repensando o uso da Inteligência Artificial devido a sua capacidade de errar. É o caso da Axon, fornecedora de câmeras de segurança para policiais nos Estados Unidos e Canadá, que recentemente baniu o uso do reconhecimento facial de suas câmeras por determinação de seu Conselho de Ética Independente ${ }^{13}$ “(...) face recognition technology should not be deployed

10 Nuffield Council on Bioethics (2018). Bioethics Briefing Note: Artificial intelligence (AI) in healthcare and research. Disponível em: http://nuffieldbioethics.org/wp-content/uploads/Artificial-Intelligence -AI-inhealthcare-and-research.pdf

11 Matt Scherer (2016). Tay the Racist Chatbot: Who is responsible when a machine learns to be evil? Disponível em: https://futureoflife.org/2016/03/27/tay-the-racist-chatbot-who-is-responsible-when-a- machine-learns-tobe-evil/

12 Apple Inc (2017). Face ID Security, página 2. Disponível em: https://www.apple.com/business/docs/site/FaceID_Security_Guide.pdf

13 Axon Inc. AI and Policing Technology Ethics Board (2019). First Report of the Axon AI \& Policing Technology Ethics Board, página 28. Disponível em: https://static1.squarespace.com/static/ 58a33e881b631bc60d4f8b31/t/5d13d7e1990c4f00014c0aeb/1561581540954/Axon_Ethics_Board_First_Rep ort.pdf 
until the technology performs with far greater accuracy and performs equally well across races, ethnicities, genders, and other identity groups" 14.

Embora seja do folclore popular que máquinas não erram, essa premissa encontra-se absolutamente infundada quando o assunto é Inteligência Artificial, portanto, faz-se necessário avançar em outro questionamento: Quem é responsável pelas decisões da Inteligência Artificial? Veremos isso mais a frente.

\section{A DIFICULDADE DE VALIDAR OS RESULTADOS FORNECIDOS POR I.A}

Um dos grandes desafios relacionados ao potencial inerente da Inteligência Artificial de proferir decisões erradas reside na dificuldade de validar os resultados e o aprendizado realizado pelos algoritmos, quanto mais eficiente o algoritmo, mais complexo e mutável ele se torna. Frequentemente utiliza-se o termo black-box ${ }^{15}$ (caixa-preta) para designar os softwares com Inteligência Artificial, mesmo que a caixa-preta seja aberta e o algoritmo lido por um cientista computacional capacitado, é pouco provável que ele possa explicar o resultado final obtido pelo software e como serão os novos resultados. O funcionamento dessas estruturas de software tenta simular as camadas de pensamento do cérebro humano, são camadas de algoritmos que podem se rearranjar e mudar seus parâmetros com base no aprendizado constante, ou seja, a cada novo dado consumido pela I.A., ela é capaz de comparar com seu banco de dados e mudar seus parâmetros de classificação e resultado.

Frequentemente entusiastas dessas novas tecnologias se perguntam qual é a diferença entre acreditar em um conhecimento de máquina e um conhecimento humano ${ }^{16}$, em outras palavras, comparam a caixa-preta da I.A. com a caixa-preta do cérebro humano. Se confiamos em um cardiologista treinado e com experiência hospitalar para realizar um diagnóstico, o que nos levaria a não acreditar numa Inteligência Artificial que também foi treinada nessa especialidade e tem o potencial de ter revisado e estudado um número de casos muito maior do que o profissional humano?

14 Em tradução livre: “(...) a tecnologia de reconhecimento facial não deve ser implantada até que funcione com mais precisão e tenha melhor desempenho com em todas as raças, etnias, gêneros e outros grupos identitários.".

15 Jordan Person (2016). When AI Goes Wrong, We Won't Be Able to Ask It Why. Disponível em: https://www.vice.com/en_us/article/vv7yd4/ai-deep-learning-ethics-right-to-explanation

16 Kate Levchuk (2018). Is Black Box Human Better Than Black Box AI? Disponível em: https://www.forbes.com/sites/cognitiveworld/2018/10/15/black-box-human-or-black-box-ai-a-talk-with-kimlarsen-deutsche-telekom/\#6befb7991932 
A capacidade do cérebro humano ainda é incomparavelmente superior à de qualquer algoritmo de Inteligência Artificial, contudo, para os especialistas, a questão não é mais se será possível atingir a capacidade humana, mas quando isso será alcançado ${ }^{17}$, estima-se que nas próximas décadas a I.A. esteja dirigindo carros, escrevendo livros e até substituindo vendedores em lojas físicas.

\section{O RISCO INERENTE DE DADOS TENDENCIOSOS USADOS PARA TREINAR A I.A}

A Inteligência Artificial só pode ser construída através de dados de treinamento, exemplificando, um algoritmo de reconhecimento de melanomas só irá conseguir efetuar essa verificação após receber um conjunto de imagens classificadas que contenha casos positivos e negativos. Após processar esses dados, o sistema poderá analisar novas imagens e definir se apresenta ou não o diagnóstico. Estes dados iniciais, chamados de dados ou amostra de treinamento, precisam ser classificados e fornecidos pelo ser humano, o que pode ocasionar a entrada de dados tendenciosos, seja porque foram mal classificados (falso positivos) ou porque carregam preconceitos de quem os produziu ou classificou. Esses dados são necessariamente históricos, assim, se a I.A. precisa estudar os casos das últimas décadas para definir a expectativa de vida e cura de um paciente, o que acontece se a diferença racial provocou uma diferença no tratamento ${ }^{18}$ e esse seja um dos fatores determinantes da morte prematura?

O algoritmo que não foi especialmente criado para discriminar acaba por realizar esse tipo de ação, agora em virtude dos dados recebidos, não de sua programação. Essa preocupação já obteve a atenção de organismos internacionais como o Fórum Econômico Mundial ${ }^{19}$, congressistas americanos ${ }^{20}$ e também da União Europeia $^{21}$. Os gigantes da tecnologia também já passaram por situações onde seus experimentos em I.A. acabaram por demonstrar preconceitos, além da Microsoft Tay oportunamente citada, o Google criou um algoritmo de

17 Katja Grace et al (2017). When Will AI Exceed Human Performance? Evidence from AI Experts. Disponível em: https://arxiv.org/abs/1705.08807

18 Tikkanem RS et al (2017). Hospital Payer and Racial/Ethnic Mix at Private Academic Medical Centers in Boston and New York City. Disponível em: https://www.ncbi.nlm.nih.gov/pubmed/28152644

19 Saheli Roy Choudhury (2018). A.I. has a bias problem that needs to be fixed: World Economic Forum. Disponível em: https://www.cnbc.com/2018/09/18/world-economic-forum-ai-has-a-bias-problem-thatneeds-to-be-fixed.html

20 Brian Resnick (2019). Yes, artificial intelligence can be racist. Alexandria Ocasio-Cortez says AI can be biased. She's right. Disponível em: https://www.vox.com/science-and-health/2019/1/23/18194717/ alexandria-ocasio-cortez-ai-bias

21 Warwick Ashford (2018). GDPR a challenge to AI black boxes. Disponível em: https://www.computerweekly.com/news/252452183/GDPR-a-challenge-to-AI-black-boxes 
classificação de sentimentos através de texto que classificava sentenças LGBT como negativas ${ }^{22}$.

A Forrester, uma das principais empresas de consultoria sobre impacto de tecnologia nos Estados Unidos publicou um estudo sobre o tema ${ }^{23}$, onde sugere que pode ser possível evitar os efeitos discriminatórios nos algoritmos e dados de treinamento através do acrônimo "FAIR: Fundamentally sound, Assessable, Inclusive, Reversible ${ }^{24}$ ". Segundo o estudo, os algoritmos devem ser construídos por dados produzidos de forma independente e distribuídos de forma idêntica (incluindo igualmente raça, cor, gênero e outros dados), devem ser passíveis de avaliação do resultado, não podem excluir ninguém do seu modelo de avaliação e devem ser reversíveis a ponto de serem usados por quem o concebeu. Sem prejuízo de ser um bom ponto de partida e produzir alguns conceitos imprescindíveis, esse conceito carrega alguns problemas, como temos demonstrado neste trabalho, já que a avaliação de resultados produzidas por I.A. apresenta uma impossibilidade a adoção total desse direcionamento.

\section{DE QUEM É A RESPONSABILIDADE QUANDO A I.A. É USADA NA TOMADA DE DECISÕES}

Neste ponto, já percebemos que a Inteligência Artificial pode ser um aliado poderoso na tomada de decisões nos cuidados em saúde, por vezes tendo resultados melhores que os profissionais de saúde, contudo, também sabemos que a Inteligência Artificial pode errar de forma grave, o que pode custar vidas e merece atenção do ponto de vista jurisdicional. Ainda não temos jurisprudência dessas situações, visto que a maioria das aplicações de Inteligência Artificial ainda está sob a guarda das equipes de saúde, mas podemos usar os casos de arguição de imperícia médica relacionados a diagnóstico computadorizado para tentar estabelecer um paralelo em busca da resposta.

A questão encontra desdobramentos espinhosos, se quando tratamos do trabalho do médico é pacífica a exigência da obrigação de meio $^{25}$, sendo raras as exceções, como a cirurgia

22 Josh Jackman (2017). Google's new artificial intelligence bot thinks gay people are bad. Disponível em: https://www.pinknews.co.uk/2017/10/26/googles-new-artificial-intelligence-bot-thinks-gay-people-are-bad/

23 Brandon Purcell (2018). The Ethics Of AI: How To Avoid Harmful Bias And Discrimination. Disponivel em: https://www.forrester.com/report/The+Ethics+Of+AI+How+To+Avoid+Harmful+Bias+And+ Discrimination/-/E-RES130023

24 Em tradução livre: FAIR: Fundamentalmente sólido, Avaliável, Inclusivo, Reversível.

25 Conforme ensina Flávio TARTUCE (pág. 155): "A obrigação de meio ou de diligência é aquela em que o devedor só é obrigado a empenhar-se para perseguir um resultado, mesmo que este não seja alcançado." TARTUCE, Flavio (2019). Direito Civil: Direito das Obrigações e Responsabilidade Civil - v. 2. 14 ed. - Rio de Janeiro, Forense. 
estética, o mesmo não tem sido aplicado quando falamos dos Serviços de Apoio Diagnóstico Terapêutico - SADT, como os exames laboratoriais e de imagem, que, embora possam ter laudos feitos por médicos, utilizam majoritariamente sistemas computacionais, seja para produzir as imagens no caso da Tomografia Computadorizada e da Ressonância Magnética, ou para quantificar dosagens em fluidos, no caso dos exames laboratoriais.

Nestes casos, tem a jurisprudência fartos exemplos ${ }^{26}$ do reconhecimento da obrigação de resultado ${ }^{27}$, ultrapassando a figura do profissional de saúde e responsabilizando a pessoa jurídica responsável pelo serviço, chegando inclusive a aplicar as regras do Código de Defesa do Consumidor. Levando em conta essa diferença de tratamento, podemos inferir que quando tratarmos de conflitos envolvendo a Inteligência Artificial nos cuidados em saúde, as obrigações de resultado ou mesmo o CDC poderão ser invocados, responsabilizando os serviços de saúde em uma situação que reconhecidamente não há acurácia perfeita, além disso, há também desdobramentos no direito de regresso. Se num laudo médico de exame ultrassonográfico o médico indicar a presença de algo que as imagens sem sombra de dúvida não contém quando exibidas a outros profissionais, ainda assim será o serviço o potencialmente culpado, mas subsistirá o direito de regresso. Já nas aplicações de Inteligência Artificial tal direito pode ser um pouco mais complexo, visto que os resultados da Inteligência Artificial não são sempre os mesmos, podendo um mesmo algoritmo fornecido por um serviço de computação apresentar, para a mesma amostra, resultados diferentes, bastando que para isso seja usada a versão original fornecida pelo desenvolvedor de software, sem ter sido treinada continuamente pelos dados durante seu uso no serviço de saúde.

Poderia então o fornecedor se eximir da culpa se na versão inicial o software afirmasse a existência de determinada doença e o resultado proferido pelo mesmo software usado no serviço de saúde afirmasse que a doença não existe? Sendo a única diferença entre os softwares que um deles foi mais utilizado (portanto tinha mais dados) que o outro?

Entendemos que é cedo para garantir uma única direção a essas perguntas, contudo, podese evitar que se chegue a este dilema não permitindo que os resultados decisórios da Inteligência Artificial sejam utilizados antes de serem avaliados e confirmados pela equipe de profissionais

26 Tribunal de Justiça de São Paulo TJ-SP - APELAÇÃO No 0176687-71.2010.8.26.0100; Tribunal de Justiça do Rio de Janeiro TJ-RJ - APELAÇÃO : APL 0075984-46.2015.8.19.0038; Tribunal de Justiça do Rio de Janeiro TJ-RJ - APELAÇÃO : APL 0004693-98.2012.8.19.0067

27 Seguindo na definição de TARTUCE (op. cit., pág.156): “na obrigação de resultado ou de fim, a prestação só é cumprida com a obtenção de um resultado, geralmente oferecido pelo devedor previamente. Aqueles que assumem obrigação de resultado respondem independentemente de culpa (responsabilidade civil objetiva) ou por culpa presumida (...)". 
do serviço de saúde, ficaria assim a Inteligência Artificial como ferramenta da equipe, que se responsabilizaria pelo resultado oferecido, podendo inclusive ser diferente do que foi oferecido pela I.A. Essa opção, de viés conservador, nos parece a melhor na maioria dos casos, garantindo a evolução e o crescimento do uso dessas tecnologias de forma segura. Contudo, veremos no tópico de Autonomia do Paciente que podem existir outros interesses com sede em direitos fundamentais nesta equação.

\section{AUTONOMIA DO PACIENTE E RESPONSABILIDADE PROFISSIONAL}

A relação entre médico e paciente vem sofrendo uma grande transformação tendo como objetivos a horizontalização da relação, a primazia da autonomia e consentimento do paciente e o direito de informação e a individualização do tratamento, entre outros. Tendo em vista esses pontos, tratamos abaixo dos direitos e garantias que surgem com a entrada da Inteligência Artificial nessa relação.

\section{RECUSA E EXIGENNCIA DE TRATAMENTO E A PERDA DE UMA CHANCE}

Considerando que o uso das ferramentas voltadas aos cuidados em saúde com Inteligência Artificial podem fornecer diagnósticos e prognósticos diretamente ao usuário, como fica a relação médico-paciente no caso do profissional divergir do resultado obtido pela I.A.?

Pode parecer questão de menor importância se colocada no âmbito das grandes cidades, onde existem vários especialistas que poderão ser consultados e, provavelmente, algum deles pode vir a concordar ou seguir inicialmente o caminho apontado pela I.A, porém, é necessário pensar justamente nas situações em cidades pequenas e longe dos grandes centros, onde a oferta de especialistas é menor, ou seja, uma I.A. treinada por resultados e pacientes do mundo inteiro apresenta um resultado a um paciente e o médico nega o atendimento, quais seriam as consequências jurídicas?

Tradicionalmente não seria necessário maior exame para garantir a supremacia do diagnóstico do médico sobre qualquer outra forma, somava-se a obrigação de meio do mesmo com o Código de Ética Médico ${ }^{28}$ que atesta como direito do médico: "II - Indicar o procedimento adequado ao paciente, observadas as práticas cientificamente reconhecidas e respeitada a legislação vigente." Contudo, após o amplo recepcionamento da teoria da perda

28 Conselho Federal de Medicina. Resolução CFM $n^{\circ}$ 1931/2009. Disponível em: http://www.portalmedico.org.br/novocodigo/integra.asp 
de uma chance no direito pátrio e da interpretação da relação médico paciente cada vez mais horizontal, podem ser encontrados na jurisprudência precedentes importantes em condenação do profissional em casos claros de perda de uma chance de cura. ${ }^{29}$

No caso de confronto entre Inteligência Artificial e o profissional médico, ressalvadas as situações de urgência, entendemos que não há ganhos para a autonomia do paciente em exigir, independente da judicialização da contenda, tratamento ou procedimento negado pelo médico, sendo caminho mais viável a exigência do mesmo ao serviço de saúde e, em via final, ao poder público. Sobre os desdobramentos no poder público, continuaremos no tópico de biopolítica.

\section{BIOPOLÍTICA, RESERVA DO POSSÍVEL E ESTRATÉGIA DE SAÚDE BÁSICA}

O alcance dos dispositivos celulares é abundante no Brasil, segundo dados da ANATEL $^{30}$, as três maiores operadoras de telefonia móvel do Brasil atendem mais de $90 \%$ dos municípios brasileiros, tendo o país mais linhas de celular ativas do que pessoas ${ }^{31}$. Pensando nisso, a utilização de ferramentas que possam garantir a esses usuários diagnóstico através de equipamentos tecnológicos já existentes, ou mesmo por novos equipamentos ${ }^{32}$, com custo acessível a administração local, pode significar sensível melhora no alcance dos cuidados básicos de saúde para toda a população, além de melhores níveis de acuidade no diagnóstico, com uma diminuição do custo geral de serviços públicos relacionados, como o Sistema Único de Saúde - SUS.

Contudo, tal nível de automatização de uma relação que é tão intrinsecamente humana como a médico-paciente pode trazer reflexos negativos no controle social das populações, sobretudo as de baixa renda e afastadas das capitais. Isso porque a Administração Pública pode

29 Danilo Leme Crespo (2017). Uma nova espécie de indenização decorrente de erro médico: a perda de uma chance. Disponível em: <https://www.migalhas.com.br/dePeso/16,MI262958,81042-Uma+nova+especie + de+indenizacao+decorrente+de+erro+medico+a+perda+de $>$

30 ANATEL - Agência Nacional de Telecomunicações (2018). Telefonia móvel - Municípios Atendidos. Disponível em: https://www.anatel.gov.br/setorregulado/component/content/article/115-universalizacao-eampliacao-do-acesso/telefonia-movel/423-telefonia-movel-municipios-atendidos

31 Pedro Hallack (2019). Número de celulares diminui, mas ainda supera quantidade de pessoas no Brasil. Disponível em: https://www.moneyreport.com.br/economia/numero-de-celulares-diminui-mas-ainda- superaquantidade-de-pessoas-no-brasil/

32 Existem equipamentos de autodiagnóstico aprovados em países como Estados Unidos, acredita-se que esses aparelhos tem custo sensivelmente menor considerando os atendimentos que é capaz de realizar do que manter uma equipe de saúde capacitada. Para mais detalhes sobre alguns desses equipamentos aprovados: https://www.mobihealthnews.com/content/autonomous-ai-diagnostic-startup-idx-scores-33m 
evocar o princípio da eficiência ${ }^{33}$ e também a teoria da reserva do possível $^{34}$ para limitar o acesso a determinados tratamentos que não estejam sendo recomendados pelo sistema automatizado, assim, as pessoas estariam condicionadas a aceitar o modo de diagnóstico definido pelo Estado para ter acesso aos serviços de saúde fornecido por este. Soma-se a isso a impossibilidade de validação dos resultados fornecidos por I.A. e temos uma forma de controle e conformação social. Tal raciocínio é semelhante ao que apresenta o pensamento de Foucault décadas atrás: "O controle da sociedade sobre os indivíduos não se opera simplesmente pela consciência ou pela ideologia, mas começa no corpo, com o corpo. Foi no biológico, no somático, no corporal que, antes de tudo, investiu a sociedade capitalista. O corpo é uma realidade biopolítica. A medicina é uma estratégia biopolítica. "35

Estamos convictos de que o acesso a ferramentas tecnológicas que possam de qualquer forma melhorar a vida das pessoas deve ser não só garantido como também incentivado, porém, a utilização destes pelo Estado para gerar qualquer tipo de conformação social deve ser restrito, garantindo para a população a oportunidade de contestar os métodos e resultados, sem que isso importe na efetivação da prestação positiva pretendida em relação ao Poder Público.

\section{NOSSO SISTEMA JURÍDICO ESTÁ PREPARADO?}

Peremptoriamente, a resposta tradicional em subverter as relações jurídicas decorrentes do uso de equipamentos diagnósticos inteligentes para o alcance do CDC é um ponto a ser reconsiderado na entrada da Inteligência Artificial no universo dos diagnósticos. Como em qualquer grande inovação, é necessário aliar a cautela com a segurança jurídica e a proteção necessária para que as novas tecnologias sejam adotadas num ambiente favorável, sob o risco de inviabilizar os estudos desta nova modalidade de diagnóstico.

As inovações legislativas que tentam acompanhar e conformar o comportamento da tecnologia são recentes, quase todas da década atual, como a Lei 12.965/2014, conhecida como

33 Na explicação de Di Pietro (2019, pág. 244): “O princípio da eficiência apresenta, na realidade, dois aspectos: pode ser considerado em relação ao modo de atuar do agente público, do qual se espera o melhor desempenho possível de suas atribuições, para lograr melhores resultados, e em relação ao modo de organizar, estruturar, disciplinar a Administração Pública, também como o objetivo de alcançar melhores resultados na prestação do serviço público." Di Pietro, Maria Sylvia Zanella. Direito Administrativo - 32 ed. - Rio de Janeiro: Forense.

34 Conforme Ingo Sarlet (2015, pág. 296): “A reserva do possível constitui, em verdade (considerada toda a sua complexidade), espécie de limite jurídico e fático dos direitos fundamentais, mas também pode atuar, em determinadas circunstâncias, como garantia dos direitos fundamentais, por exemplo, na hipótese de conflitos de direitos, quando se cuidar da invocação (...) da indisponibilidade de recursos com o intuito de salvaguardar núcleo essencial de outro direito fundamental.” SARLET, Ingo Wolfgang. A eficácia dos direitos fundamentais. 12 ed. Porto Alegre: Livraria do Advogado Editora.

35 Foucault, M. (1978). Microfísica do poder (pág. 47). Rio de Janeiro: Graal. 
Marco Civil da Internet Brasileira, a Lei 12.737/2012 que inovou em direito penal, criando o primeiro tipo penal relacionado a crimes virtuais ${ }^{36}$ e por fim a LGDP, Lei Geral de Proteção de Dados Pessoais, versão brasileira de um debate profundo ocorrido na União Européia e que deu origem alguns anos antes a GDPR: General Data Protection Regulation ${ }^{37}$.

Essencialmente, LGDP e GDPR apresentam o mesmo espírito regulatório ${ }^{38}$, garantindo ao usuário de sistemas informatizados a propriedade das informações pessoais concedidas a controladores de sistemas computadorizados, dando primazia ao consentimento do usuário para uso dos dados e garantindo que este consentimento pode ser retirado a qualquer momento. Especialmente sobre o tratamento de dados por Inteligência Artificial, aplicam-se os dispositivos relacionados a "tratamento automatizado" e "decisão automatizada", presentes na lei brasileira no Art. $20^{39}$ e na européia no Art. $22^{40}$, esses dispositivos legais garantem ao usuário o direito de solicitar a revisão de decisões tomadas unicamente por sistemas automatizados, que é o caso da Inteligência Artificial. Outro direito garantido na GDPR que também consta no parágrafo primeiro do Art. 20 da LGDP é o de receber "informações claras e adequadas a respeito dos critérios e dos procedimentos utilizados para a decisão automatizada, observados os segredos comercial e industrial. " 41

Enquanto o caput do Art. 20 da LGPD traz necessária salvaguarda para relevantes questões éticas e jurídicas levantadas no percorrer deste trabalho, a obrigação de informar presente no parágrafo primeiro do mesmo artigo pode apresentar situações complicadas ao fornecedor do software ou do serviço de saúde que o utiliza, principalmente tendo em vista a dificuldade do judiciário brasileiro de lidar com os conceitos das novas tecnologias digitais ${ }^{42}$, pois nem sempre é possível explicar de forma clara uma decisão por Inteligência Artificial.

36 Art. 154-A do Decreto-Lei 2848/40 (Código Penal Brasileiro): "Invadir dispositivo informático alheio, conectado ou não à rede de computadores, mediante violação indevida de mecanismo de segurança e com o fim de obter, adulterar ou destruir dados ou informações sem autorização expressa ou tácita do titular do dispositivo ou instalar vulnerabilidades para obter vantagem ilícita”. Disponível em: http://www.planalto.gov.br/ccivil_03/decreto-lei/del2848compilado.htm

37 European Parliament and the Council (2016). Regulation (EU) 2016/679. Disponível em: https://eurlex.europa.eu/legal-content/EN/TXT/PDF/?uri=CELEX:32016R0679

38 Richie Koch. Brazil passed the General Data Protection Law in 2018, and it will come into effect February 2020. This article examines the GDPR vs. the LGPD, how it differs, and what business owners globally need to do to prepare. Disponível em: https://gdpr.eu/gdpr-vs-lgpd/

39 Brasil. Lei 13709/2018 - Lei Geral de Proteção de Dados (LGPD). Disponível em: http://www.planalto.gov.br/ccivil_03/_ato2015-2018/2018/lei/L13709.htm

40 Op. cit. https://gdpr.eu/article-22-automated-individual-decision-making

41 Art. 20, §1 da LGDP, op. cit.

42 Fernando Martines (2017). No STF, WhatsApp alega que bloqueios ferem Marco Civil e livre iniciativa. Disponível em: https://www.conjur.com.br/2017-fev-01/stf-whatsapp-alega-bloqueios-ferem-lei- livreiniciativa 
Desta feita, a edição da LGPD traz uma base inicial para que se construa um debate e possam ser iniciados os experimentos em saúde no Brasil com Inteligência Artificial, contudo, ainda é insuficiente para regular os meios em que essa tecnologia será aplicada, seus efeitos na cobertura de planos de saúde e na judicialização dos casos onde a decisão por I.A. seja o fator determinante.

\section{CONSIDERAÇÕES FINAIS}

O universo de possibilidades da Inteligência Artificial é fantástico e apresenta novidades disruptivas para quase todos os aspectos da vida humana, contudo, como em qualquer grande inovação, não é possível prever os resultados de sua adoção em massa no desenvolvimento da sociedade. Mesmo que a maioria dos resultados sejam positivos e acompanhem melhorias na vida das pessoas, quando a Inteligência Artificial erra, temos reproduzidos os piores comportamentos da mente humana, como o racismo e a homofobia, que tanto se luta para remover da sociedade.

Tratando especificamente do tema deste trabalho, é com entusiasmo que se acompanha os resultados de sucesso de uso da Inteligência Artificial em aplicações voltadas para a saúde, tendo em vista o desafio crescente de universalização do acesso básico à saúde na sociedade de massa e em constante crescimento demográfico que vivemos.

Os riscos são muitos, este trabalho cumpre o papel de introduzir algumas importantes discussões, inclusive tentando em alguns casos discutir a matéria e fazer paralelos com a jurisprudência atual, mas está longe de esgotar o tema ou elencar todas as possibilidades de dilemas éticos e jurídicos.

Limites são necessários, experimentos científicos não estão imunes da análise ética e moral, os limites atuais da bioética talvez não sejam suficientes para esta nova modalidade de pesquisa, sendo necessário o debate de novas regras e princípios na adoção desses sistemas, sobretudo pelos poderes públicos, decisões judiciais e procedimentos médicos que podem ter efeitos permanentes.

O paciente deve ser o centro das decisões em todos os casos, autonomia e direito à informação precisam ser salvaguardados, mesmo que não seja possível explicar os resultados, é necessário informar ao menos a origem daquele resultado e garantir a possibilidade de obtenção de um resultado não automatizado, garantindo o contato humano. 


\section{REFERÊNCIAS}

Anand Avati et al. Improving Palliative Care with Deep Learning (2017). Disponível em: <https://arxiv.org/abs/1711.06402> Acesso em: 10 de abril de 2019.

ANATEL - Agência Nacional de Telecomunicações. Telefonia móvel - Municípios Atendidos (2018). Disponível em: <https://www.anatel.gov.br/setorregulado/component/ content/article/115-universalizacao-e-ampliacao-do-acesso/telefonia-movel/423-telefoniamovel-municipios-atendidos> Acesso em: 20 de junho de 2019.

ANS - Agência Nacional de Saúde. Mapa Assistencial da Saúde Suplementar 2018. Disponível em:

<http://www.ans.gov.br/images/stories/noticias/pdf/Mapa_Assistencial_2018.pdf> Acesso em: 20 de junho de 2019.

Apple Inc . Face ID Security. (2017) Disponível em: <https://www.apple.com/business /docs/site/FaceID_Security_Guide.pdf> Acesso em: 20 de junho de 2019.

Axon Inc. AI and Policing Technology Ethics Board. First Report of the Axon AI \& Policing Technology Ethics Board. (2019) Disponível em: <https://static1.squarespace.com /static/58a33e881b631bc60d4f8b31/t/5d13d7e1990c4f00014c0aeb/1561581540954/Axon_Et hics_Board_First_Report.pdf> Acesso em: 10 de junho de 2019.

Brandon Purcell. The Ethics Of AI: How To Avoid Harmful Bias And Discrimination. (2018) Disponível em: <https://www.forrester.com/report/ The+Ethics+Of+AI+How+To+ Avoid+Harmful+ Bias+And+Discrimination/-/E-RES130023> Acesso em: 10 de junho de 2019.

Brian Resnick. Yes, artificial intelligence can be racist. Alexandria Ocasio-Cortez says AI can be biased. She's right. (2019) Disponível em: <https://www.vox.com/science-and-health /2019/1/23/18194717/alexandria-ocasio-cortez-ai-bias> Acesso em: 20 de agosto de 2019.

CFM - Conselho Federal de Medicina. Resolução CFM n ${ }^{\circ}$ 1931/2009. Disponível em: <http://www.portalmedico.org.br/novocodigo/integra.asp> Acesso em: 11 de junho de 2019.

Di Pietro, Maria Sylvia Zanella. Direito Administrativo - 32 ed. 2019. Rio de Janeiro: Forense.

European Parliament and the Council. Regulation (EU) 2016/679. Disponível em: <https://eur-lex.europa.eu/legal-content/EN/TXT/PDF/?uri=CELEX:32016R0679> Acesso em 10 de junho de 2019.

Fernando Martines. No STF, WhatsApp alega que bloqueios ferem Marco Civil e livre iniciativa. (2017) Disponível em: <https://www.conjur.com.br/2017-fev-01/stf-whatsapp alega-bloqueios-ferem-lei- livre-iniciativa> Acesso em 08 de junho de 2019.

Foucault, M. Microfísica do poder. 1978. Rio de Janeiro: Graal.

H A Haenssle et al (2018). Man against machine: diagnostic performance of a deep learning convolutional neural network for dermoscopic melanoma recognition in comparison to 58 dermatologists. (2018). Disponível em: <https://academic.oup.com /annonc/article/29/8/1836/5004443> Acesso em: 10 de junho de 2019.

IEEE Spectrum. AI vs Doctors. Disponível em: <https://spectrum.ieee.org/static/ai-vsdoctors> Acesso em: 20 de junho de 2019. 
Jordan Person. When AI Goes Wrong, We Won't Be Able to Ask It Why. (2016)

Disponível em: <https://www.vice.com/en_us/article/vv7yd4/ai-deep-learning-ethics- rightto-explanation> Acesso em 10 de junho de 2019.

Josh Jackman. Google's new artificial intelligence bot thinks gay people are bad. (2017) Disponível em: <https://www.pinknews.co.uk/2017/10/26/googles-new-artificial-intelligencebot-thinks-gay-people-are-bad/> Acesso em: 20 de agosto de 2019.

Kate Levchuk. Is Black Box Human Better Than Black Box AI? (2018) Disponível em: $<$ https://www.forbes.com/sites/cognitiveworld/2018/10/15/black-box-human-or-black-box-aia-talk-with-kim-larsen-deutsche-telekom/\#6befb7991932> Acesso em 10 de junho de 2019.

Katja Grace et al. When Will AI Exceed Human Performance? Evidence from AI Experts.(2017) Disponível em: <https://arxiv.org/abs/1705.08807> Acesso em 10 de junho de 2019.

Matt Scherer. Tay the Racist Chatbot: Who is responsible when a machine learns to be evil? (2016) Disponível em: <https://futureoflife.org/2016/03/27/tay-the-racist-chatbot- whois-responsible-when-a- machine-learns-to-be-evil/> Acesso em: 20 de junho de 2019.

Nuffield Council on Bioethics. Bioethics Briefing Note: Artificial intelligence (AI) in healthcare and research. (2018) Disponível em: <http://nuffieldbioethics.org/wp-content/ uploads/Artificial-Intelligence-AI-in-healthcare-and-research.pdf> Acesso em: 10 de abril de 2019.

OECD Data. Health care use. (2017) Disponível em: <https://data.oecd.org/healthcare/ computed-tomography-ct-exams.htm> Acesso em: 20 de junho de 2019.

Pedro Hallack. Número de celulares diminui, mas ainda supera quantidade de pessoas no Brasil. (2019) Disponível em: <https://www.moneyreport.com.br/economia/numero-decelulares-diminui-mas-ainda-supera-quantidade-de-pessoas-no-brasil/>

Richie Koch. Brazil passed the General Data Protection Law in 2018, and it will come into effect February 2020. Disponível em: <https://gdpr.eu/gdpr-vs-lgpd/> Acesso em: 20 de agosto de 2019.

Saheli Roy Choudhury. A.I. has a bias problem that needs to be fixed: World Economic Forum. (2018) Disponível em: <https://www.cnbc.com/2018/09/18/world-economic- forumai-has-a-bias-problem-that- needs-to-be-fixed.html> Acesso em 20 de junho de 2019.

SARLET, Ingo Wolfgang. A eficácia dos direitos fundamentais. 12 ed. - 2015 Porto Alegre: Livraria do Advogado Editora.

TARTUCE, Flavio. Direito Civil: Direito das Obrigações e Responsabilidade Civil - v. 2. 14 ed. 2019 - Rio de Janeiro: Forense.

Tikkanem RS et al. Hospital Payer and Racial/Ethnic Mix at Private Academic Medical Centers in Boston and New York City. (2017) Disponível em:

<https://www.ncbi.nlm.nih.gov/pubmed/28152644> Acesso em 20 de agosto de 2019.

Warwick Ashford. GDPR a challenge to AI black boxes. (2018) Disponível em: <https://www.computerweekly.com/news/252452183/GDPR-a-challenge-to-AI-black-boxes> Acesso em 20 de junho de 2019. 